\title{
Characterization and analysis of heteroepitaxial growth on silicon structures
}

\author{
Ganesh Vanamu and Abhaya K. Datye \\ Department of chemical and nuclear engineering, University of New Mexico, \\ Albuquerque, NM-87131 \\ \& \\ Andy Allerman, and Saleem H. Zaidi \\ Gratings, Inc., 2700 B Broadbent Pkwy., N.E, Albuquerque, NM 87107
}

The heteroepitaxial growth of III-V compound semiconductor films such as GaN, $\mathrm{GaAs}$, and $\mathrm{Ge}$ on silicon substrates is of considerable interest for a number of optoelectronic devices including solar cells, lasers and photodetectors. For example, the growth of defect-free Ge and GaAs thin $(\sim 6-10 \mu \mathrm{m})$ films on Si substrate can potentially lead to mechanically stronger, higher efficiency, lower cost solar cells for space and terrestrial applications. Novel devices based on $\mathrm{Si}_{\mathrm{x}} / \mathrm{Ge}_{1-\mathrm{x}}$ superlattices are also of particular interest in current semiconductor research because of their potential impact on the highly developed Si-based microelectronics technology.

Due to thermal expansion and lattice coefficient mismatches, defect free growth of heteroepitaxy films beyond critical thickness, $h_{c}$ is not possible. A number of approaches including composition variation, finite area, and compliant buffer layers aimed at concentrating defects at the interface while growing high quality layers have been investigated. We have investigated epitaxial growth on micro and nanoscale $\mathrm{Si}$ structures as shown in Fig. 1. These types of structures can potentially absorb thermal expansion and lattice expansion mismatches as well as to enable removal of heteroepitaxial layers for subsequent wafer reuse. In this abstract, MOCVD growth of Ge and GaN films on micro and nanostructured silicon structures is reported. While, keeping the growth parameters constant, surface structures are varied to determine an optimum for the highest quality heteroepitaxial growth. In the preliminary work presented here, we have analyzed the cross-sections of epilayers using transmission electron microscopy (TEM) and scanning electron microscopy (SEM) to calculate the lattice constants and respective mismatches at interfaces of the heterostructures as shown in Fig. 2. In this model of calculation for the lattice constants, we used the FFT of the diffraction pattern to back out the lattice constants and find the average lattice constants at the interface and hence the lattice mismatch as shown in Fig 3. No defects are observed by the HRTEM (High resolution TEM) at the surface. The apparent lattice mismatch from the HRTEM is $0.682 \%$, as opposed to conventional $4 \%$ mismatch typical of Si and Ge. More details of structures and model interpretation will be reported at the conference. 


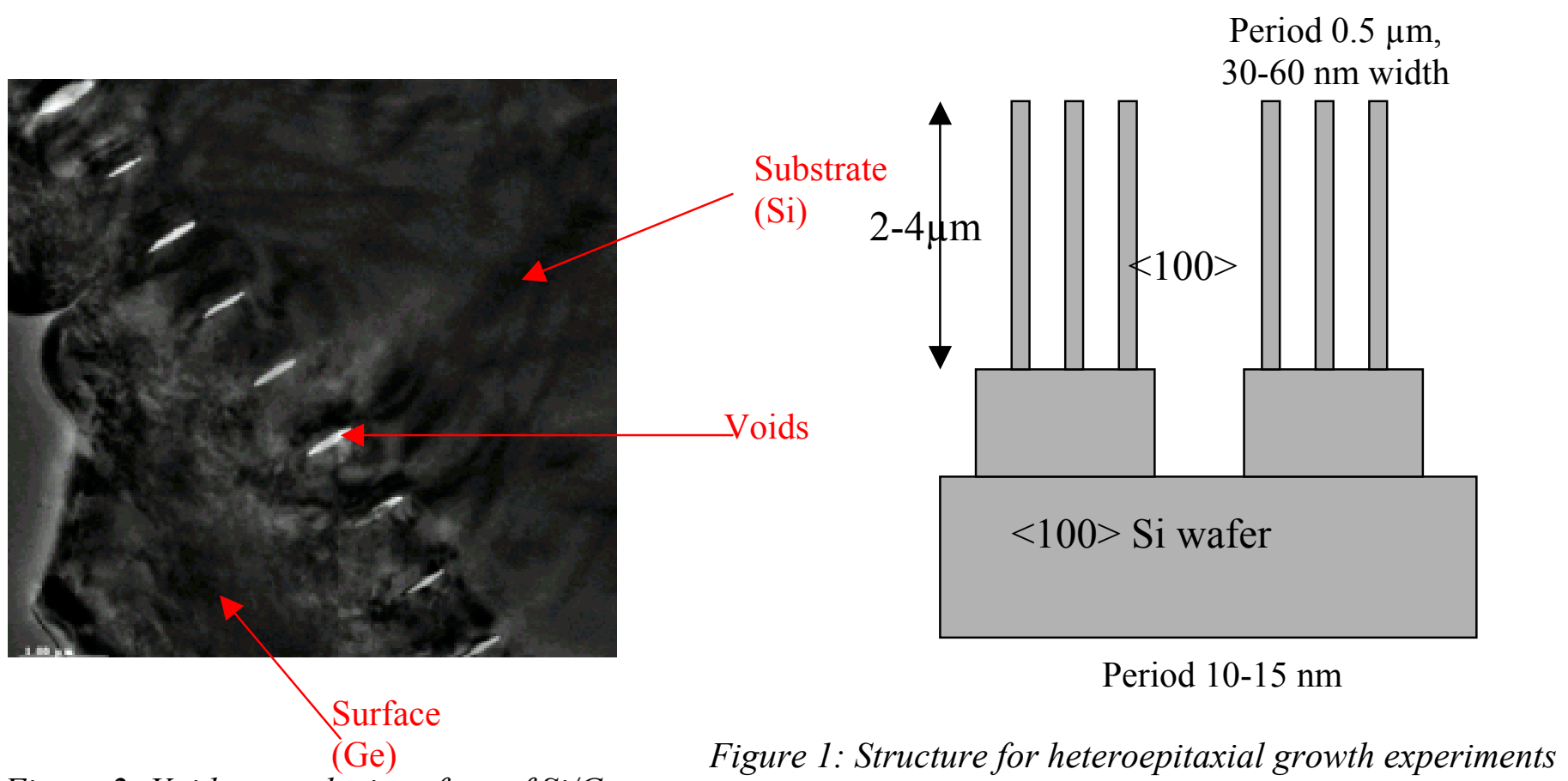

Figure 2: Voids near the interface of $\mathrm{Si} / \mathrm{Ge}$

Substrate

(Si)

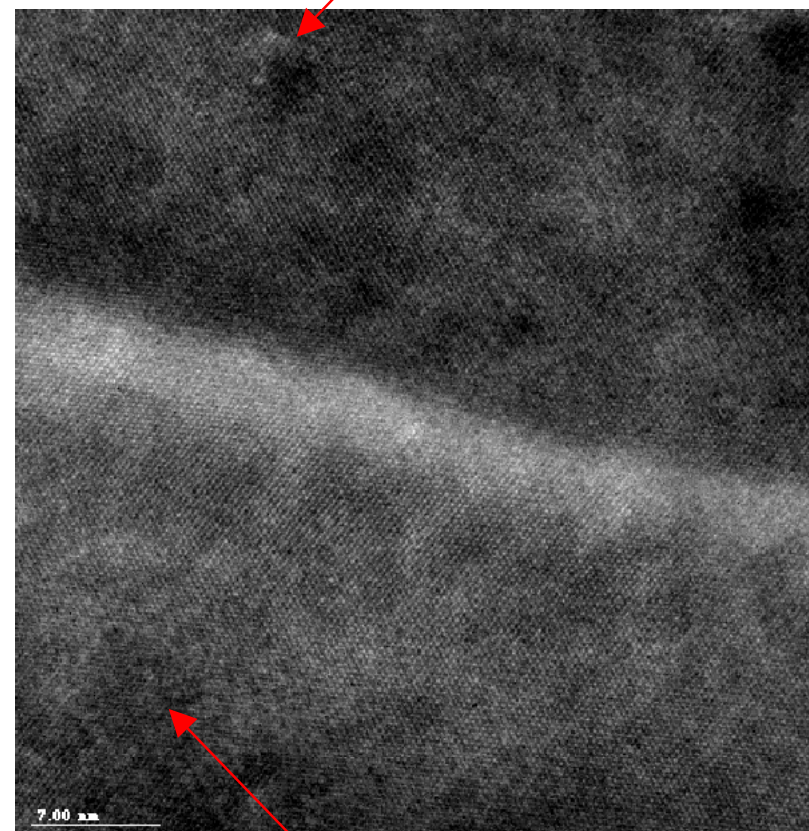

7000
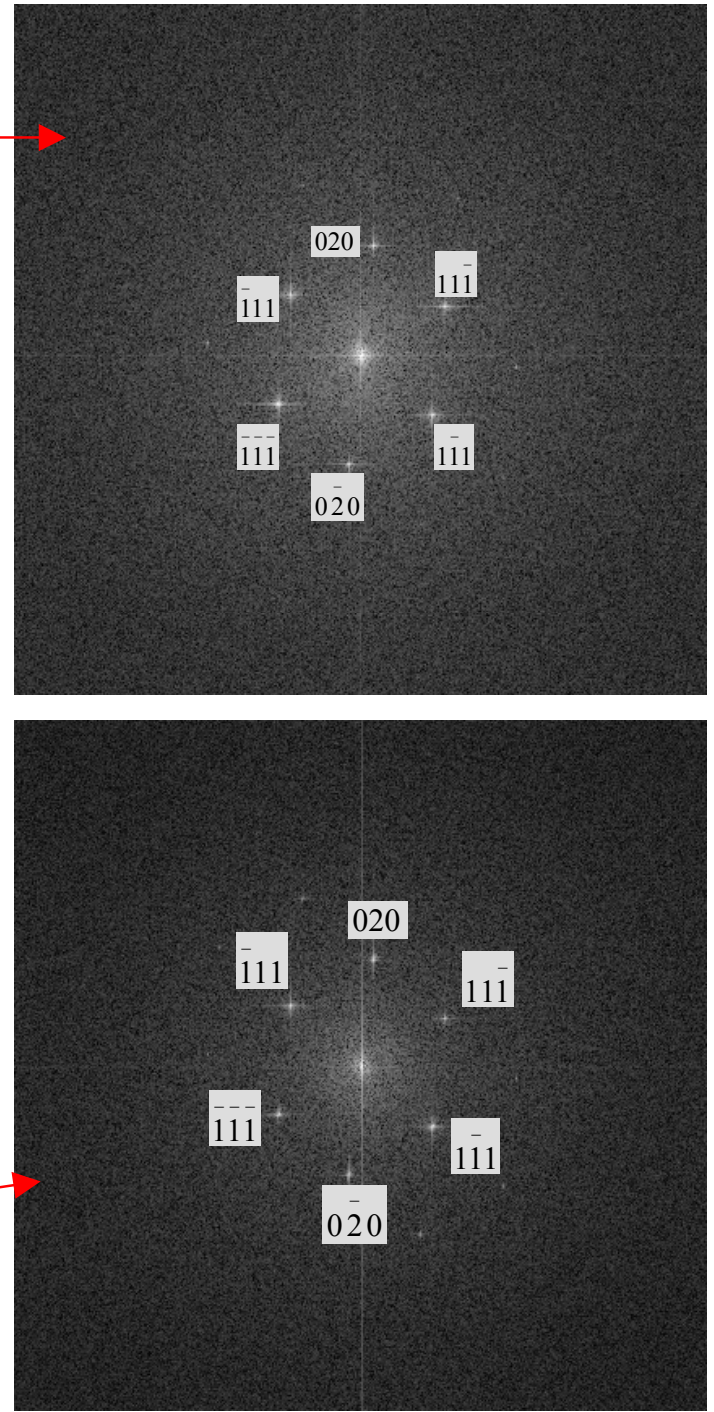

Figure 3: HRTEM pictures near the interface to calculate the lattice constants https://doi.org/10.1017/S143192760344186X Published online by Cambridge University Press 